\title{
Somatic mutations, acetylator status, and prognosis in colorectal cancer
}

\author{
J E Hardingham, W J Butler, D Roder, A Dobrovic, R B Dymock, R E Sage, \\ I C Roberts-Thomson
}

\begin{abstract}
Background-Somatic mutations in K-ras and TP53 may be associated with both acetylator status and prognosis in colorectal cancer.

Aims-To determine whether cancers with somatic mutations are more frequent in fast acetylators and whether mutations or acetylator status influence prognosis after colorectal surgery.

Patients-One hundred consecutive subjects undergoing elective surgery for colorectal cancer.

Methods-Acetylator status was determined by polymerase chain reaction (PCR) genotyping for polymorphism in the N-acetyltransferase 2 (NAT2) gene. Mutations in K-ras (codon 12) and TP53 were determined by PCR analysis using restriction enzyme digestion and single strand conformation polymorphism respectively. Survival from colorectal cancer for up to five years after diagnosis was analysed using the Kaplan-Meier product limit estimator. Cox proportional hazards regression was used to compare survival rates after adjusting for tumour stage.

Results-Mutations in K-ras and TP53 were independent of acetylator status. By log rank test, survival was significantly reduced in subjects with TP53 mutations $(p=0.003)$ but was not significantly related to acetylator status or the presence of K-ras mutations. After adjustment for tumour stage, subjects with both TP53 and K-ras mutations had a 4.2-fold case fatality (95\% confidence interval 1.5 to 11.6$)$ when compared with that of a TP53 negative reference group.

Conclusion-The presence of both TP53 and $\mathrm{K}$-ras mutations in colorectal tumours is an adverse prognostic marker which is independent of tumour stage. (Gut 1998;42:669-672)
\end{abstract}

Keywords: colorectal cancer; TP53 and K-ras mutations; acetylator status; prognosis

Numerous studies have shown that survival after surgery for colorectal cancer is influenced by the extent of local spread and metastases in lymph nodes and distant organs. More subtle histological features may have prognostic importance but some have relatively poor levels of interobserver agreement and may lose prognostic significance in multivariate analyses which include tumour stage. Other potential prognostic markers include proliferative activity, ploidy, and glycopro- tein expression but reports of their usefulness have been conflicting.

More recently, prognosis has been examined in relation to somatic mutations in various oncogenes and tumour suppressor genes including K-ras and TP53. Although mutations in TP53 appear to be associated with decreased survival, ${ }^{2-4}$ two of three studies deduced the presence of mutations on the basis of overexpression of TP53 as assessed by immunohistochemistry. Mutations in K-ras have been associated with reduced survival in some studies, ${ }^{56}$ but not others. ${ }^{78}$ Bell et al found reduced survival when tumours had both $\mathrm{K}$-ras mutations and overexpression of TP53. ${ }^{9}$

The purpose of this study was to determine the effect of K-ras and TP53 mutations on survival after colorectal surgery and to determine whether this effect was independent of the extent of tumour spread as assessed by Dukes' stage. We also examined the possibility of an association between acetylator status, somatic mutations, and prognosis since fast acetylation has been associated with an increased risk for colorectal cancer, particularly in subjects with a high intake of meat, ${ }^{10}$ and with a higher than expected frequency of K-ras mutations in tumour tissue. ${ }^{11}$

\section{Methods}

PATIENTS AND SAMPLES

Dukes' stage, acetylator status, and the presence of K-ras and TP53 mutations in tumour tissue were determined in 100 consecutive patients who underwent elective surgery for colorectal cancer between 1 March 1990 and 1 October 1994. There were 58 men and 42 women with a median age of 70 years (range 37-88). The study was approved by the institutional Ethics of Research Committee and informed consent was obtained from each patient. DNA was isolated from tumour samples obtained either fresh (84 patients) or from tissue blocks fixed in formalin and embedded in paraffin wax (16 patients).

DETECTION OF K-ras AND TP53 MUTATIONS Tumour DNA samples were analysed for K-ras mutations in codon 12 as described previously ${ }^{12}$ using primers as shown in table 1 . Briefly, a primer mediated restriction digestion assay was used to identify K-ras codon 12 mutations following polymerase chain reaction (PCR) amplification. The pattern of BstN1 digestion products on a $10 \%$ polyacrylamide gel distinguishes normal and mutant alleles.

Mutations in TP53 are clustered in the four highly conserved regions of the gene, exons 5-8. Four sets of primers (table 1) were designed to 
Table 1 Primer sequences

\begin{tabular}{ll}
\hline Primer & Sequence $5^{\prime}-3^{\prime}$ \\
\hline K-ras & \\
Sense & \\
Antisense & ACTGAATATAAACTTGTGGTAGTTGGACCT \\
TP53 exon 5 & TCAAAGAATGGTCCTGGACC \\
Sense & TCTGTCTCCTTCCTCTTCCTACA \\
Antisense & AACCAGCCCTGTCGTCTCTC \\
TP53 exon 6 & GTCCCCAGGCCTCTGATTCC \\
Sense & CCTCCTCCCAGAGACCCCCAGT \\
Antisense & \\
TP53 exon 7 & CCTCATCTTGGGCCTGTGTTATC \\
Sense & TGGCAAGTGGCTCCTGACCTGGA \\
Antisense & \\
TP53 exon 8 & CCTCTTGCTTCTCTTTTCCTATC \\
Sense & CTGCTTGense \\
NAT2 & GGTCAATCAACTTCTGTACTGGGC \\
Sense & TTGGGTGATACATACACAAGGGTT \\
Antisense &
\end{tabular}

amplify the entire coding sequence of these exons. PCR for each exon was performed in a 50 $\mu 1$ volume with $100 \mathrm{ng} \mathrm{DNA}, 200 \mu \mathrm{M}$ each of dATP, dTTP, dCTP, and dGTP, $0.2 \mu \mathrm{M}$ primers, $1 \times$ Taq buffer with $1.5 \mathrm{mM} \mathrm{MgCl}_{2}$, and 0.5 units of Taq polymerase (Boehringer Mannheim, Mannheim, Germany). Single strand conformation polymorphism (SSCP) analysis was used to detect mutations. Exon 5 products were run on a $12 \% \mathrm{~T}, 1 \% \mathrm{C}$ gel at 8 Watts for four hours while exons 6,7 , and 8 were run on a $16 \% \mathrm{~T}, 1 \% \mathrm{C}$ gel at 8 Watts for four hours. Band shifts were detected by silver staining. ${ }^{13}$

ACETYLATOR GENOTYPING

The NAT2 gene was amplified by PCR using the primer sequences (table 1 ) to yield a 704 base pair product. PCR was performed in a 50 $\mu \mathrm{l}$ volume with $100 \mathrm{ng}$ DNA, $200 \mu \mathrm{M}$ each of dATP, dTTP, dCTP, and dGTP, 100 ng primers, $1 \times$ Taq buffer with $1.5 \mathrm{mM} \mathrm{MgCl}$, and 0.5 units of Taq polymerase (Boehringer Mannheim, Mannheim, Germany). After an initial denaturation at $94^{\circ} \mathrm{C}$ for four minutes, 35 cycles were performed at $94^{\circ} \mathrm{C}$ for 25 seconds, $56^{\circ} \mathrm{C}$ for 30 seconds, and $72^{\circ} \mathrm{C}$ for 60 seconds, followed by a final extension at $72^{\circ} \mathrm{C}$ for five minutes. Various alleles for NAT2 were detected by genotyping using the restriction enzymes Kpn 1, Bam H1 with Xba 1 and Taq 1 (New England Biolabs, Beverly, Massachusetts, USA). The presence of the F1 allele in heterozygous or homozygous form indicated fast acetylation. ${ }^{14}$

MUTATIONS AND TUMOUR HISTOLOGY

The possibility of an association between mutations and histological characteristics was examined in 44 of 100 tumours, 17 with both $\mathrm{K}$-ras and TP53 mutations and 27 with neither mutation. Coded sections from tumour samples fixed in formalin and embedded in paraffin wax were stained with haematoxylin and eosin and scored for degree of differentiation (poor or non-poor), definition of margins (well or ill defined), presence of Crohn's like lymphoid aggregates ( 0 to $1+$ or 2 to $4+$ ), lymphocytic infiltration ( 0 to $1+$ or $2+$ to $4+$ ), lymphovascular invasion (present or absent), and necrosis (present or absent). ${ }^{15}$ Studies were performed by an experienced pathologist (RBD) who was unaware of the mutation status.

DATA COLLECTION AND ANALYSIS

Dates and causes of death for the 100 patients were sought through the South Australian Cancer Registry. The follow up procedures used by the Registry to ascertain and verify details from state death records have been described previously and the extent of loss to follow up and its potential effect on calculated survival rates has been shown to be very small. ${ }^{16} 17$

Survival analyses were performed using the Kaplan-Meier product limit estimator. ${ }^{18}$ Living cases were censored at 31 December 1995. Cases dying from causes other than colorectal cancer were censored at the times of their deaths. The median duration of follow up from diagnosis to death or the date of censoring (whichever came first) was approximately two years (729 days). Survival functions were compared using the log rank test. ${ }^{18}$

Cox proportional hazards regression was used to determine the relative risk of case fatality by tumour characteristic, after adjusting for Dukes' stage. ${ }^{18}$ Stage $\mathrm{D}$ was treated as the reference category and stages $\mathrm{A}, \mathrm{B}$, and $\mathrm{C}$ as dummy variables. As risk estimates for stages $\mathrm{A}$ and $B$ were similar, they were combined in the final model. While age, sex, and tumour differentiation were also entered into the initial multivariate Cox analysis, they were not predictive of survival ( $p>0.100)$ and did not condition the risk estimates for mutation status. They were thus excluded from the final model.

Stratified analyses were used to check that proportionality assumptions had been met for the multiple regression, and associations between predictor variables were examined by principal component analysis to check for multicolinearity. ${ }^{18}$ Interaction terms were also tested, but as none was found to be statistically significant, they were not retained in the model.

Frequencies of mutations in fast and slow acetylators and frequencies of histological characteristics in tumours with and without mutations were compared using the $\chi^{2}$ test (or the Fisher exact test when numbers were small) whereas comparisons of stage by acetylator status, K-ras and TP53 mutations were based on the Mann-Whitney U test.

\section{Results}

Of the 100 patients, $9 \%$ were classified as stage A, $44 \%$ as stage $\mathrm{B}, 35 \%$ as stage $\mathrm{C}$, and $12 \%$ as stage $\mathrm{D}$, with the percentage for stage $\mathrm{D}$ being lower than the corresponding $22 \%$ reported for all colorectal cases treated at teaching hospitals in Adelaide (including the Queen Elizabeth Hospital). ${ }^{17}$ Stage distribution did not vary significantly by acetylator status $(\mathrm{p}=0.350)$, K-ras mutation $(\mathrm{p}=0.452)$, or TP53 mutation $(p=0.126)$, although there was a trend towards a higher frequency of TP53 mutations in stages C and D (62\%) than in stages A and B (49\%).

Thirty five patients were genotyped as fast acetylators and 65 as slow acetylators. In the cancers, mutations in K-ras and TP53 were 
Table 2 Patient survival (mean (SEM)) from colorectal cancer according to Dukes'stage, acetylator status, and evidence of TP53 and K-ras mutations*

\begin{tabular}{|c|c|c|c|c|c|c|c|}
\hline & \multirow[b]{2}{*}{0} & \multicolumn{5}{|c|}{ Period from diagnosis $(y)$} & \multirow[b]{2}{*}{$p$ Valuet } \\
\hline & & 1 & 2 & 3 & 4 & 5 & \\
\hline All cases $(n=100)$ & 100 & $87(3)$ & $75(5)$ & $69(5)$ & $65(6)$ & $65(6)$ & - \\
\hline \multicolumn{8}{|l|}{ By stage } \\
\hline$A-B(n=53)$ & 100 & 100 & $96(3)$ & $89(6)$ & $89(6)$ & $89(6)$ & \multirow[t]{2}{*}{$<0.001$} \\
\hline C-D $(\mathrm{n}=47)$ & 100 & $71(7)$ & $52(8)$ & $47(8)$ & $39(10)$ & 39 (10) & \\
\hline \multicolumn{8}{|l|}{ Acetylator status } \\
\hline Slow $(n=65)$ & 100 & $89(4)$ & $79(5)$ & $70(7)$ & $70(7)$ & $70(7)$ & \multirow[t]{2}{*}{0.261} \\
\hline Fast $(\mathrm{n}=35)$ & 100 & $83(6)$ & $68(9)$ & $68(9)$ & 59 (11) & 59 (11) & \\
\hline \multicolumn{8}{|l|}{$\mathrm{K}$-ras mutation } \\
\hline No $(n=65)$ & 100 & $89(4)$ & $78(6)$ & $70(7)$ & $70(7)$ & $70(7)$ & \multirow[t]{2}{*}{0.148} \\
\hline Yes $(n=35)$ & 100 & $82(7)$ & $70(8)$ & $66(8)$ & $57(11)$ & $57(11)$ & \\
\hline \multicolumn{8}{|l|}{ TP53 mutation } \\
\hline No $(n=45)$ & 100 & $91(4)$ & $88(5)$ & $82(7)$ & $82(7)$ & $82(7)$ & \multirow[t]{2}{*}{0.003} \\
\hline Yes $(n=55)$ & 100 & $83(5)$ & $65(7)$ & $58(8)$ & $50(10)$ & $50(10)$ & \\
\hline \multicolumn{8}{|c|}{ TP53 positive cases only } \\
\hline \multicolumn{8}{|c|}{$\mathrm{K}$-ras mutation } \\
\hline No $(n=38)$ & 100 & $87(6)$ & $70(8)$ & $64(10)$ & $64(10)$ & $64(10)$ & \multirow[t]{2}{*}{0.046} \\
\hline Yes $(n=17)$ & 100 & $76(10)$ & $53(12)$ & $46(12)$ & $23(17)$ & $23(17)$ & \\
\hline
\end{tabular}

Values are given as percentages.

^Kaplan-Meier product limit estimator; date of censoring, 31 December 1995.

†Log rank test.

present in $35 \%$ and $55 \%$ respectively; $17 \%$ had both mutations. Of those with TP53 mutations, 15 were located in exon 5 , nine in exon 6,16 in exon 7 , and 15 in exon 8 . There were no significant associations between acetylator status and K-ras mutations $(\mathrm{p}=0.742)$, acetylator status, and TP53 mutations $(\mathrm{p}=0.916)$, or K-ras mutations and TP53 mutations $(\mathrm{p}=0.343)$.

As table 2 shows, survival for up to five years from diagnosis was lower for more advanced stages $(p<0.001)$ and in the presence of TP53 mutations $(p=0.003)$. Survival tended to be lower when TP53 mutations were present, both for earlier stages $(\mathrm{A}$ and $\mathrm{B})$ and later stages $(\mathrm{C}$ and D) (table 3).

There was no statistically significant difference in survival of patients who were fast acetylators compared with slow acetylators, or in patients with $\mathrm{K}$-ras mutations compared with those without (table 2). Among 55 patients with TP53 mutations, those positive for K-ras mutations had a lower survival than negative patients (five year survival $23 \%$ versus $64 \%, \mathrm{p}=0.046$ ). By comparison, $\mathrm{K}$-ras mutations were not predictive of survival among patients who were negative for a TP53 mutation $(\mathrm{p}=0.720)$.

After adjusting for tumour stage, survival was not associated with acetylator status $(p=0.895)$ or K-ras mutation $(p=0.273)$. However, survival was lower in the presence of TP53 mutations $(p=0.033)$. Table 4 shows that patients positive for both TP53 and K-ras mutations had a particularly high risk of case fatality, with a relative risk of 4.2 (confidence interval 1.5 to 11.6 ) when compared with a reference category of TP53 negative patients.
Table 4 Relative risk of case fatality (95\% confidence interval) from colorectal cancer according to Dukes'stage and presence of TP53 and K-ras mutations *

\begin{tabular}{ll}
\hline & Relative risk \\
\hline Stage & 1.00 \\
D (reference) & $0.31(0.12$ to 0.81$)$ \\
C & $0.05(0.02$ to 0.18$)$ \\
A or B & 1.00 \\
Mutation status & $2.01(0.71$ to 5.67$)$ \\
TP53 negative (reference) & \\
TP53 positive/K-ras negative & $4.16(1.49$ to 11.64$)$ \\
TP53 positive/K-ras positive & \\
\hline
\end{tabular}

${ }^{\star}$ Cox proportional hazards regression; date of censoring, 31 December 1995.

A histological comparison of tumours with and without both K-ras and TP53 mutations revealed that those with both mutations were more likely to have ill defined margins (100\% versus $74 \%, p=0.032)$ and showed a tendency towards a higher frequency of poor differentiation $(71 \%$ versus $44 \%, p=0.090)$ and $1 y m-$ phovascular invasion $(59 \%$ versus $30 \%$, $\mathrm{p}=0.055)$. The frequency of Crohn's like lymphoid aggregates $(76 \%$ versus $81 \%, p=0.716)$, lymphocytic infiltration $2+$ to $4+(59 \%$ versus $37 \%, p=0.158)$, and necrosis $(65 \%$ versus $56 \%, \mathrm{p}=0.548$ ) was similar in the two groups.

\section{Discussion}

In this study, the frequency of mutations in cancer tissue and the distribution of fast and slow acetylators were similar to those in previous reports. ${ }^{10-21}$ Furthermore, the data corroborate previous reports which indicate that mutations in TP53 and $\mathrm{K}$-ras are independent events in which neither is a prerequisite for the other. ${ }^{9}$ Specifically, the frequency of TP53

Table 3 Patient survival (mean (SEM)) from colorectal cancer by Dukes'stage according to evidence of TP53*

\begin{tabular}{|c|c|c|c|c|c|c|c|c|}
\hline \multirow[b]{2}{*}{ Stage } & \multirow[b]{2}{*}{ TP53 mutation } & \multirow[b]{2}{*}{0} & \multicolumn{5}{|c|}{ Period from diagnosis $(y)$} & \multirow[b]{2}{*}{$p$ Valuet } \\
\hline & & & 1 & 2 & 3 & 4 & 5 & \\
\hline \multirow[t]{2}{*}{$\mathrm{A}-\mathrm{B}$} & No $(n=27)$ & 100 & 100 & 100 & 100 & 100 & 100 & \multirow[t]{2}{*}{0.038} \\
\hline & Yes $(n=26)$ & 100 & 100 & $91(6)$ & $78(10)$ & $78(10)$ & $78(10)$ & \\
\hline \multirow{2}{*}{$\mathrm{C}-\mathrm{D}$} & No $(n=18)$ & 100 & $77(10)$ & $70(11)$ & $61(13)$ & $61(13)$ & $61(13)$ & \multirow[t]{2}{*}{0.033} \\
\hline & Yes $(n=29)$ & 100 & $68(9)$ & $40(10)$ & $40(10)$ & $20(15)$ & $20(15)$ & \\
\hline
\end{tabular}

^Kaplan-Meier product limit estimator; date of censoring, 31 December 1995. 
mutations in tumours with and without K-ras mutations was not significantly different $(p=0.343)$ at $49 \%$ and $58 \%$ respectively.

Although fast acetylation has been associated with an increased risk for colorectal cancer in some studies, ${ }^{102}$ we were unable to confirm a previous report indicating that cancers in fast acetylators had a higher frequency of K-ras mutations. ${ }^{11}$ In the present study, the frequency of K-ras mutations in fast and slow acetylators was $37 \%$ and $34 \%$ respectively while TP53 mutations in fast and slow acetylators had a frequency of $54 \%$ and $55 \%$ respectively. Although a relation between carcinogen exposure and particular gene mutations is an attractive hypothesis, it is of interest that colorectal tumours in rats induced by heterocyclic amines (carcinogens activated by acetylation) rarely showed TP53 or K-ras mutations but did have mutations in the rat apc gene. ${ }^{23}$

The most important observation in this study was that five year survival after diagnosis was impaired, independent of tumour stage, in those patients where cancers had both TP53 and K-ras mutations. As in previous reports, the effect of mutations in TP53 appeared to be greater than that of mutations in K-ras. ${ }^{2-9}$

Differences in the relation between acquired mutations and prognosis between this and other reports may reflect differences in statistical power due to variable sample size, or differences in case selection, methods for detection of mutations, or follow up tracing and attribution of cause of death. In the present study, there was a trend towards higher survival proportions in the absence of K-ras mutations $(p=0.148)$ and when the acetylator status was slow $(p=0.261)$. The probability of not achieving statistical significance at the $5 \%$ level for differences of this magnitude would have exceeded $80 \%$, given the number of subjects. ${ }^{24}$ In relation to the detection of mutations, mutations in the ras oncogene were sought only for the most common mutation (K-ras, codon 12). Mutations in codons 13 and 61 have been identified but are rare (less than $10 \%$ frequency ${ }^{19}$ and have not been examined in relation to prognosis. For mutations in TP53, overexpression of a TP53 product appears to be a reliable indicator of mutation but false positives and negatives may occur. ${ }^{25}$ The use of a PCR based technique should be more sensitive and specific but would not detect a small number of mutations (less than $5 \%$ ) that occur outside exons $5-8$.

Reasons for the effects of somatic mutations on prognosis may include a relation between gene mutations and "aggressiveness" as assessed by histological characteristics, frequency of DNA aneuploidy, and responses to adjuvant or palliative therapies. In relation to histological characteristics, the limited data reported in this study corroborate a previous report ${ }^{9}$ in showing that tumours with both $\mathrm{K}$-ras and TP53 mutations are more likely to have poorly defined margins and show a trend towards a higher frequency of other adverse prognostic features including poor differentiation and lymphovascular invasion. Furthermore, another study has shown a higher frequency of TP53 mutations in aneuploid rather than diploid tumours, ${ }^{26}$ the former being a potential prognostic marker for poor survival. ${ }^{1}$ In relation to palliative therapy, TP53 mutations have been associated with resistance to chemotherapy ${ }^{27}$ whereas K-ras mutations were independent of responses to chemotherapy when given for known metastatic disease. $^{8}$

This work was presented at the Plenary Session, Australian Gastroenterology Week, Adelaide, September 1996. We thank Mrs Jing-Xian $\mathrm{Mi}$ and Mrs $\mathrm{R}$ Goland for technical and secretarial assistance.

1 Cohen AM, Minsky BD, Schilsky RL. Cancer of the colon. In: De Vita VT, Hellman S, Rosenberg SA, eds. Cancer. Principles and practice of oncology. Philadelphia: LippincottRaven Publishers, 1997:1144-97.

2 Starzynska T, Bromley M, Ghosh A, et al. Prognostic significance of p 53 overexpression in gastric and colorectal carcicance of p53 overexpression in gastric

3 Nathanson SD, Linden MD, Tender P, et al. Relationship among p53, stage, and prognosis of large bowel cancer. Dis Colon Rectum 1994:37:527-34.

4 Goh H-S, Yao J, Smith DR. p53 point mutation and survival in colorectal cancer patients. Cancer Res 1995;55:5217-21.

5 Miyahara M, Saito T, Kaketani K, et al. Clinical significance of ras p21 overexpression for patients with an advanced colorectal cancer. Dis Colon Rectum 1991;34:1097-102.

6 Benhattar J, Losi L, Chaubert P, et al. Prognostic significance of K-ras mutations in colorectal carcinoma. Gastroenterology 1993;104:1044-8.

7 Laurent-Puig P, Olschwang S, Delattre O, et al. Survival and acquired genetic alterations in colorectal cancer. Gastroenterology 1992;102:1136-41.

8 Markowitz S, Hines JD, Lutterbaugh J, et al. Mutant K-ras oncogenes in colon cancers do not predict patient's chemotherapy response or survival. Clin Cancer Res 1995; 1:441-5.

9 Bell SM, Scott N, Cross D, et al. Prognostic value of p53 overexpression and c-Ki-ras gene mutations in colorectal cancer. Gastroenterology 1993;104:57-64.

10 Roberts-Thomson IC, Ryan P, et al. Diet, acetylator phenotype, and risk of colorectal neoplasia. Lancet 1996;347: $1372-4$

11 Oda Y, Tanaka M, Nakanishi I. Relation between the occurrence of K-ras gene point mutations and genotypes of polymorphic $\mathrm{N}$-acetyltransferase in human colorectal carcinomas. Carcinogenesis 1994;15:1365-9.

12 Hardingham JE, Kotasek D, Farmer B, et al. ImmunobeadPCR: a technique for the detection of circulating tumor cells using immunomagnetic beads and the polymerase chain reaction. Cancer Res 1993;53:3455-8.

13 Bassam BJ, Caetano-Anolles G, Gresshoff PM. Fast and sensitive silver staining of DNA in polyacrylamide gels. Anal Biochem 1991;196:80-3.

14 Hickman D, Sim E. N-acetyltransferase polymorphism: comparison of phenotype and genotype in humans. Biochem Pharmacol 1991;42:1007-14.

15 Shepherd NA. Pathological prognostic factors in colorectal cancer. In: Kirkham N, Lemoine NR, eds. Progress in pathology. Vol 2. Edinburgh: Churchill Livingstone, 1995: pathology.

16 Bonett A, Roder D, Esterman A. Cancer case-survival rates for South Australia: a comparison with US rates and a preliminary investigation of time trends. Med F Aust 1988;148: $556-9$.

17 South Australian Cancer Registry. Epidemiology of cancer in South Australia. Incidence, mortality and survival, 1977 to 1996. Incidence and mortality, 1996. Adelaide: Openbook Publishers, 1997.

18 Armitage P, Berry G. Statistical methods in medical research. Oxford: Blackwell Scientific Publications, 1988:421-39.

19 Bos JL, Fearon ER, Hamilton SR, et al. Prevalence of ras gene mutations in human colorectal cancers. Nature 1987; 327:293-7.

20 Vogelstein B, Fearon ER, Hamilton SR, et al. Genetic alterations during colorectal-tumor development. $N$ Engl $\mathcal{F}$ Med 1988;319:525-32.

21 Hollstein M, Sidransky D, Vogelstein B, et al. P53 mutations in human cancers. Science 1991;253:49-53.

22 Minchin RF, Kadlubar FF, Ilett KF. Role of acetylation in colorectal cancer. Mutation Res 1993;290:35-42.

23 Kakiuchi H, Watanabe M, Ushijima T, et al. Specific 5'-GGGA-3'to 5'-GGA-3' mutation of the Apc gene in rat colon tumors induced by 2-amino-1-methyl-6phenylimidazo[4,5-b]pyridine. Proc Natl Acad Sci USA 1995;92:910-4

24 Freedman LS. Tables of the number of patients required in clinical trials using the log rank test. Stat Med 1982;1:121-9.

5 Wynford-Thomas D. P53 in tumour pathology: can we trust immunocytochemistry? f Pathol 1992;166:329-30.

26 Remvikos Y, Laurent-Puig P, Salmon RJ, et al. Simultaneous monitoring of p53 protein and DNA content of colorectal adenocarcinomas by flow cytometry. Int f Cancer 1990;45: 450-6.

27 Lowe SW, Ruley HE, Jacks T, et al. P53-dependent apoptosis modulates the cytotoxicity of anticancer agents. Cell 1993;74:957-67. 